\title{
Exceptional Two-Photon Absorption in Alkynylruthenium-Gold Nanoparticle Hybrids
}

\author{
Cristóbal Quintana, Mahbod Morshedi, Huan Wang, Jun Du, Marie P. Cifuentes, and Mark G. \\ Humphrey*
}

Research School of Chemistry, Australian National University, Canberra, ACT 2601, Australia.

\begin{abstract}
Ruthenium alkynyl "star" complexes with tri(2-thienyl)-, tris(1,2,3-triazolyl)-, or triphenyl-benzene cores stabilize gold nanoparticles (AuNPs). Cyclic voltammetry, transmission electron microscopy, molecular modeling, dynamic light scattering, $\mathrm{X}$-ray photoelectron spectroscopy, and energy-dispersive X-ray spectroscopy studies are consistent with ca. 5 trithienyl- or triazolylbenzene-cored star complexes decorating the exterior of each AuNP. The ca. $2.5 \mathrm{~nm}$ diameter (by TEM) trithienylbenzene-cored gold nanoparticle hybrids are significantly less absorbing than classical Brust nanoparticles stabilized by 1-dodecanethiol; with femtosecond pulsed radiation, they exhibit exceptionally strong saturable absorption and two-photon absorption across the visible range and into the NIR region $\left(3,000,000 \mathrm{GM}\right.$ at $500 \mathrm{~nm}, 46,000 \mathrm{GM}$ at $\left.750 \mathrm{~nm} ; 1 \mathrm{GM}=10^{-50} \mathrm{~cm}^{4} \mathrm{~s}_{\text {photon }}{ }^{-1}\right)$.
\end{abstract}

KEYWORDS. Gold nanoparticles, inorganic materials, metal alkynyl complexes, nonlinear optics, organometallics.

Nonlinear absorption (NLA) processes in molecular and nano-materials are of great interest due to their potential applications in optical limiting, imaging, medicine, sensors, and telecommunications. ${ }^{1-4}$ Amongst the diverse array of materials that have been explored, plasmonic structures (e.g. silver and gold nanoparticles) have been shown to afford NLA-active materials $;{ }^{5}$ these provide field enhancement from the localized surface plasmon resonance (LSPR) at the metal-dielectric interface. Gold nanoparticles (AuNPs), in particular, are straightforward to prepare and their plasmonic excitations occur on very short timescales, affording fast NLA (ns to fs regime) that is suitable for eye and sensor protection. ${ }^{6,7}$ In order to improve NLA performance, AuNPs stabilized by agents and matrices such as thiolates, ${ }^{8-13}$ amines, ${ }^{14}$ coordination complexes, ${ }^{15}$ polymers, ${ }^{16,17}$ and carbon allotropes ${ }^{18}$ have been examined, but there have been very few ultrafast (ps-fs regime) NLA studies of such AuNP hybrid materials, a notable exception being the report of outstanding two-photon absorption (2PA) cross-sections at $800 \mathrm{~nm}$ for AuNP-thiolate hybrids $\left(\mathrm{Au}_{25}\right.$ : 427,000 GM, Au $2406: 3,452,000 \mathrm{GM}$, determined by fs two-photon excited fluorescence (2PEF); 1 GM (GöppertMayer $\left.)=10^{-50} \mathrm{~cm}^{4} \mathrm{~s}_{\text {photon }}{ }^{-1}\right) \cdot{ }^{10}$ Despite this promising result, wavelength-dependence studies of the nonlinear optical (NLO) properties of AuNP hybrids across the visible and near-infrared (NIR) regimes are scarce, the only example (to the best of our knowledge) being a spectral dependence study of AuNPcaptopril hybrids over the range 550-1100 nm (maximal 2PA cross-section 25,000 GM at $550 \mathrm{~nm}$, determined by fs Z-scan; captopril $=(2 S)-1-[(2 S)-2-$ methyl-3sulfanylpropanoyl]pyrrolidine-2-carboxylic acid). ${ }^{9}$

Dendrimers, rods, and stars (zero-generation dendrimers) containing trans- $\left[\mathrm{Ru}\left(\mathrm{C}_{2} \mathrm{R}\right) \mathrm{X}(\mathrm{dppe})_{2}\right]$ units $\left(\mathrm{X}=\mathrm{Cl}, \mathrm{C}_{2} \mathrm{R}\right.$; dppe $=$ 1,2-bis(diphenylphosphino)ethane) display much greater multi-photon absorption (MPA) activity than their purely organic analogues, ${ }^{19-22}$ with strong 2PA, three-photon absorption and (in some cases) four-photon absorption in the
NIR region; intense metal-to-ligand charge-transfer (MLCT) in the visible region and $\mathrm{d} \pi-\mathrm{p} \pi$ orbital overlap between the ruthenium and the alkynyl ligands are key contributors to the outstanding NLO merit of these organometallic complexes. ${ }^{23,24}$ AuNP-organometallic dendrimer hybrids have shown promise for applications in catalysis, ${ }^{25,26}$ sensing, ${ }^{27,28}$ and energy storage, ${ }^{27}$ but there are thus far no studies of the NLO properties of AuNP-organometallic dendrimer hybrids, particularly 2PA spectral-dependency studies, despite the abovementioned strong MPA performance of organometallic dendrimers/stars and the significant improvement in NLA that can accrue from coupling molecular materials to AuNPs. Herein, we report the syntheses, characterization, and 2PA spectral dependence studies of the first examples of metal alkynyl star-AuNP hybrids; the resultant ultra-small organometallic-capped gold nanoparticles exhibit long-term stability, solubility, reversible redox activity, good optical transparency, and exceptional 2PA performance.

The new organometallic stars Ru4, Ru5, and Ru6 that were targeted to stabilize the ultra-small AuNP hybrids consist of a central aromatic unit connected to AuNP-stabilizing moieties (1,4-(1,2,3-triazolyl $)^{27-29}$ or 2,5-thienyl $\left.{ }^{30,31}\right)$ or 1,4-phenylenyl (employed as control), which are in turn linked through phenylethynyl $\pi$-bridges to trans $-\left[\mathrm{Ru}(\mathrm{C} \equiv \mathrm{CPh})(\mathrm{dppe})_{2}\right]$ termini. The individual "arms" of the stars $(\mathbf{R u 1}, \mathbf{R u 2}$, and $\mathbf{R u 3})$ were also prepared, to afford insight into the importance of the branched composition, while a purely organic 1,4-(1,2,3triazolyl)-containing star $\quad 1,3,5-\left(\mathrm{PhC} \equiv \mathrm{C}-1,4-\mathrm{C}_{6} \mathrm{H}_{4}-1,2,3-\right.$ $\left.\mathrm{C}_{2} \mathrm{~N}_{3} \mathrm{H}-4\right)_{3} \mathrm{C}_{6} \mathrm{H}_{3}$ (Ru0, an analogue of $\mathbf{R u 4}$ ) was synthesized to benchmark the significance of the trans-[Ru(dppe $\left.)_{2}\right]$ unit (Figure 1); the organic analogue of Ru5 could not be purified due to insolubility and so was not pursued further. The trans$\left[\mathrm{Ru}(\mathrm{C} \equiv \mathrm{CPh})(\text { dppe })_{2}\right]$ termini impart several advantages: good thermal stability and high solubility in conventional organic solvents, crucial considerations for processing purposes, and reversible redox activity coupled to strong changes in linear and 
NLO properties, which can afford a means to switch such properties. ${ }^{23,24}$ The syntheses of the complexes, their precursors, and the organic star Ru0 are described in full in the Supporting Information (Schemes S1-S7). The new complexes and organic compounds were characterized by various spectroscopic methods (Figures S1-S44), cyclic voltammetry studies were carried out on all complexes (Figures S89-S94), and singlecrystal X-ray diffraction studies were undertaken on Ru1 and Ru2 (Figures S113 and S114). The new complexes and Ru0 were then assayed for stabilization of AuNPs. Our initial attempt to prepare AuNP-organometallic star hybrids employing the one-phase (Turkevich) $\operatorname{method}^{32}$ was unsuccessful, the ${ }^{31} \mathrm{P}$ NMR spectrum suggesting the formation and then decomposition of vinylidene species (Figure S104). We therefore subsequently pursued a modified Brust methodology, ${ }^{33}$ which afforded the target nanomaterials as dark-purple (Ru4-AuNP, Ru6-AuNP) and dark-green (Ru5AuNP) powders. The star complexes Ru4, Ru5, and Ru6 are essential for the stabilization of the AuNPs, a black insoluble residue being obtained in the absence of a stabilizing agent or when using the star "arm" complexes Ru1, Ru2 or Ru3 or the organic star Ru0 as stabilizing agents (Figure S106).

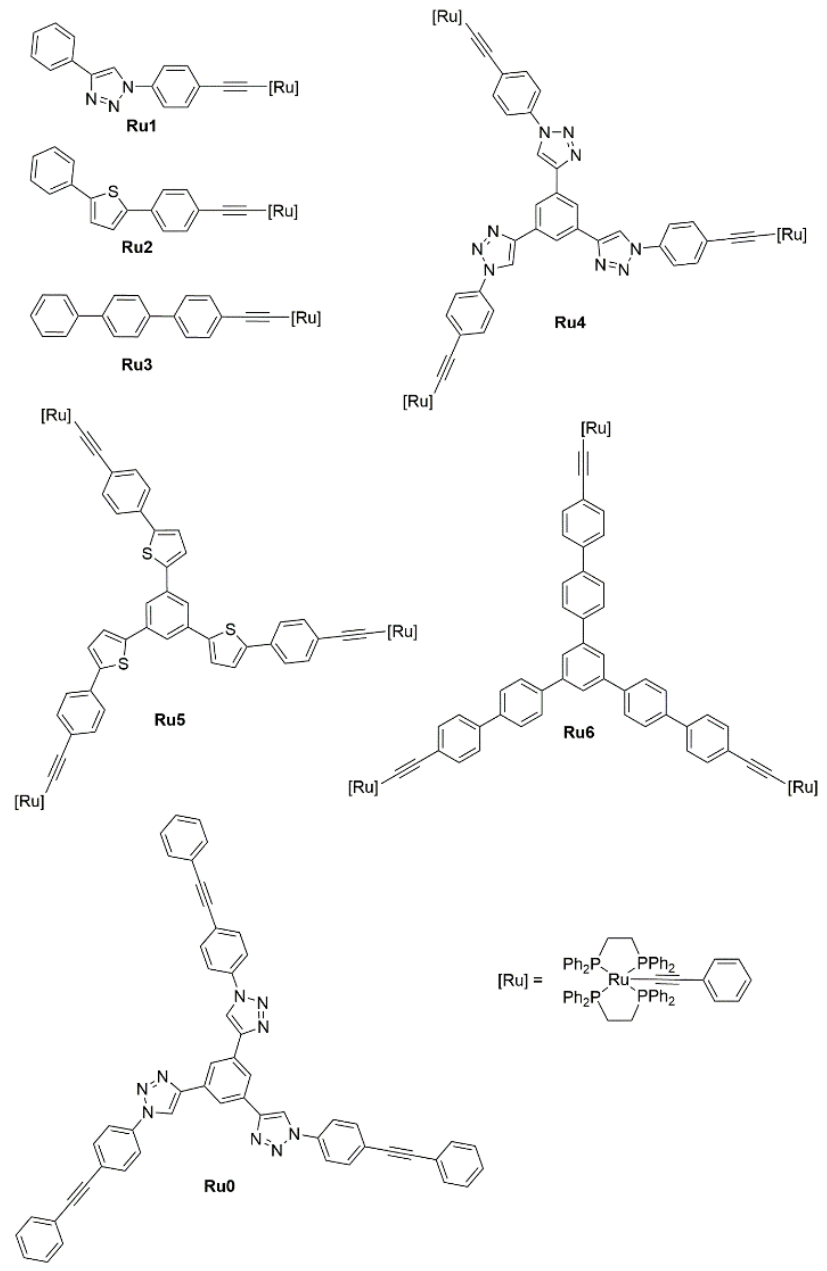

Figure 1. Linear and star compounds employed in this study.

The hybrids Ru4-AuNP, Ru5-AuNP, and Ru6-AuNP display a singlet in their ${ }^{31} \mathrm{P}$ NMR spectra at ca. $53 \mathrm{ppm}$ (Figures $\mathrm{S} 45-\mathrm{S} 47)$ in a similar location to their alkynyl complex precursors (Figures S30, S35, and S42). The ${ }^{31} \mathrm{P}$ NMR resonances of trans-[Ru(dppe $\left.)_{2}\right]$-containing species are diagnostic of the ligated-metal environment; the spectra of Ru5-AuNP were invariant over seven days, indicative of its highly robust nature in solution (Figure S105). Cyclic voltammetry studies were undertaken for all organometallic complexes and AuNP hybrids (Figures S89-S97, Tables S1 and S2). In all cases, a metal-centered reversible oxidation process was observed, similar to those observed in other trans$\left[\mathrm{Ru}\left(\mathrm{C}_{2} \mathrm{R}\right)\left(\mathrm{C}_{2} \mathrm{R}^{\prime}\right)(\text { dppe })_{2}\right]$ complexes, ${ }^{34}$ and with the data consistent with progression from monometallic to trimetallic complex and then AuNP hybrid resulting in little change in ease of oxidation. The UV-Vis spectra of Ru4, Ru5, and Ru6 contain strong MLCT transitions at low energy in all cases, with a sizeable red shift being observed for the thienylene-containing Ru5 and a blue-shift being seen for the triazolylene-containing Ru4 compared to the phenylene-containing example Ru6 (Figure S48, S50, S53). The UV-Vis spectra of the AuNP hybrids show the presence of the LSPR bands, suggestive of the successful preparation of AuNPs, together with bands corresponding to $\pi-\pi^{*}$ and MLCT transitions at the same wavelengths as those of the free complexes Ru4, Ru5, and Ru6 (Figures S49, S51, and S54), a similar spectroscopic "additivity" to that of poly(thiophene)-AuNP hybrids. ${ }^{30} \mathrm{UV}-\mathrm{V}$ is spectroscopy kinetic studies revealed no significant change in the LSPR bands over $24 \mathrm{~h}$ (Figure S98), suggesting that the surfaces of the AuNPs are rapidly passivated by the star complexes following addition of $\mathrm{NaBH}_{4}$.

Studies to suggest the size and composition of the hybrids and the location of the star complexes were then undertaken. Transmission electron microscopy (TEM) studies revealed a relatively narrow size distribution of the AuNP hybrids (Table S3). The smallest nanoparticles were observed for Ru5-AuNP $(2.5 \pm 0.7 \mathrm{~nm})$ and Ru4-AuNP $(2.7 \pm 0.7 \mathrm{~nm})$, with Ru6-AuNP $(4.9 \pm 1.4 \mathrm{~nm})$ significantly larger in size (Figures S99-S101). The results from the qualitative elemental analysis techniques energy-dispersive spectroscopy (Figures S107-S109) and X-ray photoelectron spectroscopy (Figures S110-S112) are consistent with the anticipated elemental composition of phosphineligated ruthenium alkynyl AuNP hybrids. The catalytic activity of the hybrids for the reduction of 4-nitrophenol was assessed, and the results compared to the catalytic activity of $n$ dodecanethiol-coated AuNPs (ndt-AuNP), prepared locally by the classical Brust-Schiffrin method. ${ }^{33}$ The results of the catalyses showed low conversion for Ru5-AuNP (37\%), followed by ndt-AuNP (55\%), Ru6-AuNP (77\%), and Ru4AuNP (80\%) under the standard conditions employed (Table S6). The catalytic efficiency correlates inversely with the thiophene vs. 1,2,3-triazole binding strength to the gold surface $^{35}$ for the similar-sized nanoparticles Ru5-AuNP and Ru4-AuNP, while we presume that the large molecular diameter of Ru5 blocks access to the gold surface to a greater extent than the linear $n$-dodecanethiol ligand of ndt-AuNP; the lower activity of Ru5-AuNP compared to ndt-AuNP is consistent with the stars being located exterior to the AuNPs rather than serving as cores to nucleate AuNP formation. Solvatochromic studies revealed hypsochromic shifts in the position of the LSPR bands with decreasing solvent polarity. Ru6-AuNP shows the strongest solvatochromic shift, consistent with the strongest interaction of the AuNPs with the environment, and therefore with the weakest interaction with its star component (Figure S55), and again consistent with the stars being located on the periphery of the hybrids. To further confirm the location of the star complexes in the hybrids, Ru4AuNP was dissolved in toluene, stirred for $1 \mathrm{~h}$, and then an 
excess of $n$-dodecanethiol was added to the solution; the size distribution of the resultant Ru4-AuNP-ndt obtained from TEM micrographs showed no significant variation compared to that of Ru4-AuNP (Figure S102), indicating that Ru4-AuNP does not undergo aggregation and that Ru4 is located on the surface of the nanomaterial. ${ }^{31} \mathrm{P}$ NMR monitoring of the addition of Ru5 to a solution of Ru4-AuNP suggested rapid displacement of Ru4 (concomitant with formation of a black insoluble residue), again consistent with the stars being located on the periphery of the nanoparticles. Dynamic light scattering (DLS) studies of the hybrids were undertaken (Table S4), the polydispersity indexes being consistent with highly monodisperse nanoparticles (PDI $<0.1$ ). The DLS study of Ru5-AuNP suggests a hydrodynamic diameter of $3.0 \pm 0.5 \mathrm{~nm}$. The structural study of Ru2 suggests that the "width" of Ru5 is ca. $1.2 \mathrm{~nm}$, so the maximum distance across a Ru5-AuNP with uniform single star coverage would correspond to $2.5+(2 \times 1.2)$ $=$ ca. $5 \mathrm{~nm}$. The DLS study is therefore consistent with nonuniform single star coverage of the AuNP by the star complexes, with the observed hydrodynamic diameter falling between the TEM $2.5 \mathrm{~nm}$ diameter (corresponding to the AuNP) and the calculated $5 \mathrm{~nm}$ diameter for uniform single star coverage, and therefore likely resulting from a combination of AuNP antipode pairs (i) each with a star, (ii) one with a star, and (iii) neither with a star. Modelling to understand the relationship between the size distribution of the hybrids and the molecular size of the star complexes (underpinned by the crystal structure studies of Ru1 and Ru2, from which geometric parameters of the star arms were taken) revealed that the peripheries of Ru4, Ru5 and Ru6 define triangles with calculated average area and diameter (length from base to corner) of $600 \AA^{2}$ and $31 \AA$, respectively (Figures S115-S117), and these must be distributed about the surface of the nanoparticles. Space-filling representations of our suggested structures for Ru5-AuNP, with four or five molecules of star Ru5 around an AuNP, are given in Figure S118 (the hybrids are stabilized by a combination of strong Au-S and weaker Au... $\pi$ aryl interactions; increasing the number of stars beyond five results in unrealistically long Au-S distances. This leaves regions of free gold that we assume are stabilized by interaction with solvent).

The nonlinear refraction and nonlinear absorption properties of the new complexes and the AuNP hybrids were assessed by the Z-scan technique over the spectral range 500-900 nm, and employing low repetition rate ca. 130 fs pulses to minimize contributions from excited-state absorption (ESA); no measurable NLA activity was observed for Ru1 and Ru6, and so detailed studies of the NLO responses of these compounds were not pursued. Open-aperture and closed-aperture scans were undertaken, affording simultaneous evaluation of the spectral dependencies of the absorptive and refractive components of the hyperpolarizability. The NLO data are displayed in Figures S119-S138, with the real parts of the thirdorder nonlinear susceptibility $\left(\gamma_{\text {real }}\right)$ negative across much of the spectral range for most of the complexes and hybrids, and with negative maximal values at wavelengths corresponding to maximal positive values of the imaginary components of the third-order nonlinear susceptibility $\left(\gamma_{\text {imag }}\right)$, as expected from a nonlinear Kramers-Kronig relationship.

The maximal values of the (effective) 2PA cross-sections of the complexes and hybrids are listed in Table 1. The complexes exhibit long-wavelength onsets of absorption at 430 (Ru1), 480 (Ru2), 460 (Ru3), 580 (Ru4), 525 (Ru5), and 480 nm (Ru6) and comparatively modest maximal values of $2 \mathrm{PA}$ crosssection at optically transparent wavelengths. In contrast to expectations based on the lower aromatic stabilization energy and greater $\pi$-electron density of thiophene compared to benzene, the incorporation of thienyl units does not enhance the nonlinearity, the 2PA cross-section of Ru5 at $800 \mathrm{~nm}$ (580 GM, Figure S125) being similar to that of the oligo(phenyleneethynylene)-based star 1,3,5-\{trans$\left.\left[\mathrm{Ru}(\mathrm{C} \equiv \mathrm{CPh})(\mathrm{dppe})_{2}\left(\mathrm{C} \equiv \mathrm{CC}_{6} \mathrm{H}_{4}-4-\mathrm{C} \equiv \mathrm{C}\right)\right]\right\}_{3} \mathrm{C}_{6} \mathrm{H}_{3}(700 \mathrm{GM}) .{ }^{36}$

The NLA behavior of the AuNP hybrids broadly corresponds to saturable absorption (SA) at short wavelengths and 2PA at longer wavelengths. Strong SA is observed for the AuNP hybrids at wavelengths corresponding to the LSPR bands (530$580 \mathrm{~nm}$ ) (Figures S127, S130, S133), with maxima at $550 \mathrm{~nm}$. $\mathrm{SA}$ is common for plasmonic nanoparticles of the group 11 elements at LSPR wavelengths, due to the electronic structure being more accurately described by band theory rather than molecular orbital theory, and the resultant ease with which the valence electrons can be pumped to higher excited states through ESA; as a result, the effective 2PA cross-section can be very large (e.g. $\left((3.0 \pm 0.7) \times 10^{6}\right.$ GM @ 500 nm for Ru5AuNP, $(1.1 \pm 0.1) \times 10^{6}$ GM @ 550 nm for the Brust standard ndt-AuNP). At longer wavelengths, the observed NLA of the AuNP hybrids is 2PA in nature, with the absolute values very large for materials measured under fs conditions. [We note that the linear absorption in the region $750-800 \mathrm{~nm}$, though much less than that observed at 500-550 nm, is appreciable, and so contributions from ESA cannot be discounted; we therefore use the label "effective 2PA cross-section"]. The maximal value of the effective 2PA cross-section for ndt-AuNP is 23,000 GM at $750 \mathrm{~nm}$, but its residual linear optical absorption is significant $\left(\varepsilon_{750}=53,000 \mathrm{M}^{-1} \mathrm{~cm}^{-1}\right)$. In contrast, the triazolyl-, thienyl-, and phenylene-stabilized examples exhibit large to exceptionally large 2PA values in regions of considerably lower absorption (Ru4-AuNP: $4000 \mathrm{GM}$ at $750 \mathrm{~nm}, \varepsilon_{750}=10,000 \mathrm{M}^{-1} \mathrm{~cm}^{-1}$; Ru5AuNP: $46,000 \mathrm{GM}$ at $750 \mathrm{~nm}, \varepsilon_{750}=8000 \mathrm{M}^{-1} \mathrm{~cm}^{-1}$; Ru6AuNP: $130,000 \mathrm{GM}$ at $\left.800 \mathrm{~nm}, \varepsilon_{800}=32,000 \mathrm{M}^{-1} \mathrm{~cm}^{-1}\right)$.

Different approaches can be employed to compare the $2 \mathrm{PA}$ efficiency of disparate materials, ${ }^{37,38}$ scaling the $2 \mathrm{PA}$ crosssection by the formula weight $\left(\sigma_{2} / \mathrm{FWt}\right)$ being the most widelyaccepted. The average formula weights of the AuNP hybrids have been estimated by considering the TEM diameters to correspond to gold-only AuNP spheres, and the AuNP hybrids to consist of these spheres with partial monolayers of stars, the extent of star coverage being estimated from the DLS studies. The resultant estimated average formula weights are given in Table 1 . There are clearly significant uncertainties associated with this formula weight estimation, but the Z-scan studies themselves afford data with substantial uncertainties, ${ }^{39,40}$ and the resultant weight-scaled data have some utility. Using this estimation, exceptional weight-scaled 2PA efficiencies are obtained for Ru5-AuNP $\left(\sigma_{2} / \mathrm{FWt} 0.48 \pm 0.09 \mathrm{GM} \mathrm{mol} \mathrm{g}^{-1}\right.$ at 750 $\mathrm{nm})$ and Ru6-AuNP $\left(\sigma_{2} / \mathrm{FWt} 0.22 \pm 0.05 \mathrm{GM} \mathrm{mol} \mathrm{g}^{-1}\right.$ at 800 $\mathrm{nm})$. The former exhibits outstanding formula weight-scaled effective 2PA efficiency at shorter wavelengths (Ru5-AuNP: $31 \pm 7 \mathrm{GM} \mathrm{mol} \mathrm{g}^{-1}$ at $500 \mathrm{~nm}$ ), significantly larger than the largest extant data for gold nanorods (aspect ratio 3.4, $8 \mathrm{GM}$ mol g-1 at $550 \mathrm{~nm}$ ), gold nanoshells (120 nm silica nanospheres, $10 \mathrm{~nm}$ gold shells, $2.6 \mathrm{GM} \mathrm{mol} \mathrm{g} \mathrm{g}^{-1}$ at $\left.600 \mathrm{~nm}\right)$, and $\mathrm{Au}_{25}$ (captopril) ${ }_{18}$ nanoclusters $\left(2.7 \mathrm{GM} \mathrm{mol} \mathrm{g}^{-1}\right.$ at $\left.550 \mathrm{~nm}\right){ }^{9}$ Finally, we note that, in contrast to ruthenium alkynyl complexes, the AuNP hybrids exhibit fluorescence when excited at the high-energy end of the visible region (Figures 
S139-S143). The quantum yields are low, but the resultant action cross-sections are amongst the largest thus far for gold nanoclusters or nanoparticles (Table S7). research. M.G.H. obtained funding for the research. M.P.C. and M.G.H. supervised the other four researchers. C.Q., M.P.C. and M.G.H. interpreted the research results and wrote the manuscript.

Table 1. Linear optical and nonlinear optical absorption cross-section maxima for Ru1, Ru2, Ru3, Ru4, Ru5, Ru6, Ru4-AuNP, Ru5AuNP, Ru6-AuNP and ndt-AuNP. ${ }^{\mathrm{a}}$

\begin{tabular}{|c|c|c|c|c|}
\hline 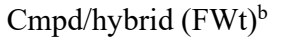 & $\lambda_{\max }[\varepsilon]$ & $\sigma_{2}\left[\lambda_{\max }, \varepsilon\right]$ & $\sigma_{2} / \mathrm{FWt}\left[\lambda_{\max }\right]$ & Size distribution $(\mathrm{nm})$ \\
\hline Ru1 (1243) & $364[2.7]$ & $\mathrm{c}$ & - & - \\
\hline Ru2 (1258) & $404[3.8]$ & $1250 \pm 180[500,0]$ & $0.99 \pm 0.15[500]$ & - \\
\hline Ru4 (3574) & $368[14.1]$ & $650 \pm 160[750,0]$ & $0.18 \pm 0.04[750]$ & - \\
\hline Ru5 (3618) & 428 [10.9] & $3500 \pm 580[530,0.7]$ & $0.96 \pm 0.16[530]$ & - \\
\hline Ru4-AuNP $(119,560)$ & 530 [21.2] (LSPR) & $4000 \pm 700[750,1.0]$ & $0.033 \pm 0.006[750]$ & $2.7 \pm 0.7$ \\
\hline Ru5-AuNP $(95,060)$ & 535 [15.4] (LSPR) & $\begin{array}{l}3.0 \times 10^{6} \pm 7.3 \times 10^{5}[500,21.3] \\
49,000 \pm 6300[610,5.7] \\
46,000 \pm 8100[750,0.8]\end{array}$ & $\begin{array}{l}31 \pm 7[500] \\
0.52 \pm 0.07[610] \\
0.48 \pm 0.09[750]\end{array}$ & $2.5 \pm 0.7$ \\
\hline
\end{tabular}

[a] Measurements in DMF solvent; $\lambda_{\max }$ in $\mathrm{nm},[\varepsilon]$ in $10^{4} \mathrm{M}^{-1} \mathrm{~cm}^{-1}, \sigma_{2}$ values in GM $\left(1 \mathrm{GM}=10^{-50} \mathrm{~cm}^{4} \mathrm{~s}\right) ;[\mathrm{b}] \mathrm{FWt}$ in $\mathrm{g} / \mathrm{mol}$; [c] 2PA not observed; [d] measurements in toluene solvent. LSPR = localized surface plasmon absorption maximum.

In summary, thienyl-containing alkynylruthenium stabilizers such as Ru5 are highly effective for the preparation of AuNP hybrids. The trans- $\left[\mathrm{Ru}\left(\mathrm{C}_{2} \mathrm{R}\right)\left(\mathrm{C}_{2} \mathrm{R}^{\prime}\right)(\mathrm{dppe})_{2}\right]$ unit and the star composition ensure solubility, stabilization, and the facile isolation of the hybrids, and enhance the NLO performance. The resulting ultra-small functional hybrids exhibit reversible redox activity, exceptional 2PA cross-sections and excellent action cross-sections at technologically-important wavelengths.

\section{ASSOCIATED CONTENT}

Supporting Information. Detailed experimental procedures for the syntheses and characterization and nonlinear optical data are reported in the Supporting Information.

\section{AUTHOR INFORMATION}

\section{Corresponding Author}

* E-mail: mark.humphrey@anu.edu.au.

\section{Author Contributions}

The chemical synthesis and spectroscopic characterization were carried out by C.Q. The electrochemical studies were carried out by C.Q. and M.M. J.D. carried out the single-crystal X-ray diffraction studies. The catalysis, TEM, DLS, EDS, and analysis of the XPS were carried out by C.Q. The molecular modelling was undertaken by M.M. The nonlinear absorption studies were carried out by C.Q, M.M., and H.W. M.G.H. and C.Q. devised the

\section{ACKNOWLEDGMENT}

We thank the Australian Research Council for support of this work (grant number DP170100408). C.Q. thanks Becas Chile (CONICYT) for financial support in the form of a $\mathrm{PhD}$ scholarship. H.W. and J.D. thank the China Scholarship Council and the Australian National University for CSC-ANU scholarships. The authors acknowledge the facilities, and the scientific and technical assistance, of the Australian Microscopy \& Microanalysis Research Facility and the Centre of Advanced Microscopy at the Australian National University.

\section{ABBREVIATIONS}

2PA, two-photon absorption; 2PEF, two-photon excited fluorescence; AuNPs, gold nanoparticles; captopril, (2S)-1-[(2S)-2methyl-3-sulfanylpropanoyl]pyrrolidine-2-carboxylic acid; DLS, dynamic light scattering; DMF, dimethylformamide; dppe, 1,2bis(diphenylphosphino)ethane; ESA, excited-state absorption; $\mathrm{GM}$, Göppert-Mayer units $=10^{-50} \mathrm{~cm}^{4} \mathrm{~s}_{\text {photon }}{ }^{-1}$; LSPR, localized surface plasmon resonance; MLCT, metal-to-ligand chargetransfer; MPA, multi-photon absorption; MW, molecular weight; ndt-AuNP, $n$-dodecanethiol-coated AuNPs prepared by the BrustSchiffrin method; NIR, near-infrared; NLA, nonlinear absorption; NLO, nonlinear optical; NMR, nuclear magnetic resonance; SA, saturable absorption; TEM, transmission electron microscopy; UVVis, ultraviolet-visible.

\section{REFERENCES}


(1) Tutt, L. W.; Boggess, T. F. Prog. Quant. Electron. 1993, 17, 299-338.

(2) Kim, H. M.; Cho, B. R. Chem. Rev. 2015, 115, 5014-5055.

(3) Chen, G.; Roy, I.; Yang, C.; Prasad, P. N. Chem. Rev. 2016, 116, 2826-2885.

(4) Dini, D.; Calvete, M. J. F.; Hanack, M. Chem. Rev. 2016 , 116, 13043-13233.

(5) Kamat, P. V. J. Phys. Chem. B 2002, 106, 7729-7744.

(6) Mathews, S. J.; Kumar, S. C.; Giribabu, L.;. Rao, S. V Mater. Lett. 2007, 61, 4426-4431.

(7) François, L.; Mostafavi, M.; Belloni, J.; Delouis, J.-F.;

Delaire, J.; Feneyrou, P. J. Phys. Chem. B 2000, 104, 6133-6137.

(8) Philip, R.; Chantharasupawong, P.; Qian, H.; Jin, R.; Thomas, J. Nano Lett. 2012, 12, 4661-4667.

(9) Olesiak-Banska, J.; Waszkielewicz, M.; Matczyszyn, K.; Samoc, M. RSC Adv. 2016, 6, 98748-98752.

(10) Ramakrishna, G.; Varnavski, O.; Kim, J.; Lee, D.; Goodson, T. J. Am. Chem. Soc. 2008, 130, 5032-5033.

(11) Ding, S.-J.; Nan, F.; Yang, D.-J.; Liu, X.-L.; Wang, Y.-L.; Zhou, L.; Hao, Z.-H.; Wang, Q.-Q. Sci. Rep. 2015, 5, 9735.

(12) Russier-Antoine, I.; Bertorelle, F.; Vojkovic, M.; Rayane, D.; Salmon, E.; Jonin, C.; Dugourd, P.; Antoine, R.; Brevet, P.-F. Nanoscale 2014, 6, 13572-13578.

(13) Hamanaka, Y.; Okada, N.; Fukagawa, K.; Nakamura, A.; Tai, Y.; Murakami, J. J. Phys. Chem. C 2012, 116, 10760-10765..

(14) Polavarapu, L.; Venkatram, N.; Ji, W.; Xu, Q. H. ACS Appl. Mater. Interfaces 2009, 1, 2298-2303.

(15) Moreau, J.; Lux, F.; Four, M.; Olesiak-Banska, J.; Matczyszyn, K.; Perriat, P.; Frochot, C.; Arnoux, P.; Tillement, O.; Samoc, M.; Ponterini, G.; Roux, S.; Lemercier, G. Phys. Chem. Chem. Phys. 2014, 16, 14826-14833.

(16) Sun, W.; Dai, Q.; Worden, J. G.; Huo, Q. J. Phys. Chem. B 2005, 109, 20854-20857.

(17) Oh, E.; Fatemi, F. K.; Currie, M.; Delehanty, J. B.; Pons, T.; Fragola, A.; Lévêque-Fort, S.; Goswami, R.; Susumu, K.; Huston, A. L.; Medintz, I. L. Part. Part. Syst. Charact. 2013, 30, 453-466.

(18) Zheng, C.; Chen, W.; Huang, Y.; Xiao, X.; Ye, X. RSC Adv. 2014, 4, 39697-39703.

(19) Roberts, R. L.; Schwich, T.; Corkery, T. C.; Cifuentes, M. P.; Green, K. A.; Farmer, J. D.; Low, P. J.; Marder, T. B.; Samoc, M.; Humphrey, M. G. Adv. Mater. 2009, 21, 2318-2322.

(20) Zhao, H.; Simpson, P. V.; Barlow, A.; Moxey, G. J.; Morshedi, M.; Roy, N.; Philip, R.; Zhang, C.; Cifuentes, M. P.; Humphrey, M. G. Chem. Eur. J. 2015, 21, 11843-11854.
(21) Simpson, P. V.; Watson, L. A.; Barlow, A.; Wang, G.; Cifuentes, M. P.; Humphrey, M. G. Angew. Chem. Int. Ed. 2016, 55, 2387-2391.

(22) Schwich, T.; Barlow, A.; Cifuentes, M. P.; Szeremeta, J.; Samoc, M.; Humphrey, M. G. Chem. Eur. J. 2017, 23, 8395-8399.

(23) Dalton, G. T.; Cifuentes, M. P.; Petrie, S.; Stranger, R.; Humphrey, M. G.; Samoc, M. J. Am. Chem. Soc. 2007, 129, $11882-$ 11883.

(24) Green, K. A.; Cifuentes, M. P.; Corkery, T. C.; Samoc, M.; Humphrey, M. G. Angew. Chem. Int. Ed. 2009, 48, 7867-7870.

(25) Daneshvar, A.; Moghadam, M.; Tangestaninejad, S.; Mirkhani, V.; Mohammadpoor-Baltork, I.; Khalili, A. Organometallics 2016, 35, 1747-1755.

(26) Friederici, M.; Angurell, I.; Seco, M.; Rossell, O.; Llorca, J. Dalton Trans. 2011, 40, 7934-7940.

(27) Wang, Y.; Salmon, L.; Ruiz, J.; Astruc, D. Nature Commun. 2014, 5, 3489-1 - 3489-12.

(28) Astruc, D.; Daniel, M.-C.; Ruiz, J. Chem. Commun. 2004, 2637-2649.

(29) Boisselier, E.; Diallo, A. K.; Salmon, L.; Ornelas, C.; Ruiz, J.; Astruc, D. J. Am. Chem. Soc. 2010, 132, 2729-2742.

(30) Jung, Y. J.; Govindaiah, P.; Park, T.-J.; Lee, S. J.; Ryu, D. Y.; Kim, J. H.; Cheong, I. W. J. Mater. Chem. 2010, 20, 9770-9774.

(31) Zhai, L.; McCullough, R. D. J. Mater. Chem. 2004, 14, 141143.

(32) Turkevich, J.; Stevenson, P. C.; Hillier, J. Disc. Faraday Soc. 1951, 11, 55-75.

(33) Brust, M.; Walker, M.; Bethell, D.; Schiffrin, D. J.; Whyman, R. J. Chem. Soc., Chem. Commun. 1994, 801-802.

(34) Gao, B.; Mazur, L. M.; Morshedi, M.; Barlow, A.; Wang, H.; Quintana, C.; Zhang, C.; Samoc, M.; Cifuentes, M. P.; Humphrey, M. G. Chem. Commun. 2016, 52, 8301-8304.

(35) Häkkinen, H. Nature Chem. 2012, 4, 443-455.

(36) Cifuentes, M. P.; Powell, C. E.; Morrall, J. P.; McDonagh, A. M.; Lucas, N. T.; Humphrey, M. G.; Samoc, M.; Houbrechts, S.; Asselberghs, I.; Clays, K.; Persoons, A.; Isoshima, T. J. Am. Chem. Soc. 2006, 128, 10819-10832.

(37) Perez-Moreno, J.; Kuzyk, M. G. Adv. Mater. 2011, 23, 1428

(38) Schwich, T.; Cifuentes, M. P.; Gugger, P. A.; Samoc, M.; Humphrey, M. G. Adv. Mater. 2011, 23, 1433-1435.

(39) Samoc, M.; Samoc, A.; Luther-Davies, B.; Humphrey, M. G.; Wong, M.-S. Opt. Mater. 2002, 21, 485-488.

(40) Morrall, J. P.; Dalton, G. T.; Humphrey, M. G.; Samoc, M. Adv. Organomet. Chem. 2008, 55, 61-136. 
Bis(diphosphine)ruthenium alkynyl "star" complexes stabilize 2-3 nm diameter gold nanoparticles (AuNPs) under Brust synthesis conditions. The resultant trithienylbenzene-cored ruthenium alkynyl star-AuNP hybrids are redox-active, very stable, and highly soluble, and exhibit good transparency, exceptional two-photon absorption cross-sections, and excellent "action" cross-sections at technologically important wavelengths.
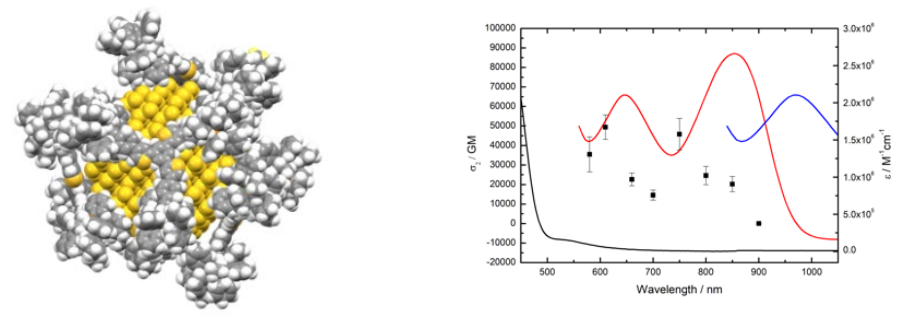